\title{
Application of statistical design for the optimization of dextranase production by a novel fungus isolated from Red Sea sponge
}

\author{
Nayera A. M. Abdelwahed · Eman Fadl Ahmed • \\ Eman W. El-Gammal • Usama W. Hawas
}

Received: 14 September 2013/ Accepted: 20 November 2013/Published online: 17 December 2013

(C) The Author(s) 2013. This article is published with open access at Springerlink.com

\begin{abstract}
Marine endophytic fungi isolated from Red Sea organisms were screened for the production of dextranase enzyme. The most potent isolate was from the Red Sea sponge Callyspongia spp. and was selected for identification. The $18 \mathrm{~S}$ rRNA amplification for phylogenetic study revealed that the isolate was highly related to Aspergillus flocculosus strain NRRL 5224 by $99 \%$. Medium composition and culture conditions for dextranase production were optimized by response surface methodology. A significant influence of dextran, yeast extract, $\mathrm{K}_{2} \mathrm{HPO}_{4}$, $\mathrm{NaNO}_{3}, \mathrm{NaCl}, \mathrm{MgSO}_{4} .7 \mathrm{H}_{2} \mathrm{O}$ and culture requirements such as incubation time, inoculum size, medium volume and inoculum age on dextranase production was evaluated by Plackett-Burman design. The most significant factors were further optimized using Box-Behnken design. The model predicted a dextranase activity of $438.15 \mathrm{U} / \mathrm{ml}$ when dextran concentration, medium volume and incubation time were $2.1 \mathrm{~g} / \mathrm{l}, 52.47 / 250 \mathrm{ml}$ flask and $80.48 \mathrm{~h}$, respectively. Verification of the model showed that dextranase production of $440 \mathrm{U} / \mathrm{ml}$ was observed under the optimal condition confirming the validity of the model.
\end{abstract}

Keywords Dextranase - Fungi - Optimization · Response surface methodology

N. A. M. Abdelwahed ( $\square)$ · E. F. Ahmed · E. W. El-Gammal Pharmaceutical Industries Division, Chemistry of Natural and Microbial Products Department, National Research Centre, El-Behouth St, Dokki, Cairo 12311, Egypt

e-mail: niarawahed@yahoo.com

U. W. Hawas

Marine Chemistry Department, Faculty of Marine Sciences, King Abdulaziz University, P. O. Box 80207, Jeddah 21589, Kingdom of Saudi Arabia

\section{Introduction}

Dextranase [(1-6)- $\alpha$-D-glucan 6-glucanohydrolases] hydrolyzes the 1-6 glycosidic linkage in dextran chain (Sankpal et al. 2001). Since the first reports on Cellvibrio fulva dextranase in the 1940's, more than 1,500 scientific papers and more than 100 patents have been issued on dextranhydrolyzing enzymes found in a number of microbial groups, fungi being the most important commercial source of dextranase (Khalikova et al. 2005). Dextranase enzyme has important industrial applications: in medicine, dextranases are used for partial hydrolysis of native dextran in the preparation of blood substitutes (Molodova et al. 1980). Also, the enzyme depolymerizes various troublesome microbial dextran deposits in teeth and prevents tooth decay. It is also used for preparing low molecular weight dextran and cytotoxic dextran conjugate and dextranase is showed as an enhancer of antibiotic activity in endocarditis (Khalikova et al. 2005; Eggleston and Monge 2004; Marotta et al. 2002; Mghir et al. 1994). Since polysaccharides interfere in sugar manufacturing process in addition to loss of sucrose resulting in heavy loss to sugar factories (Priyanka and Santosh 2011), thus for improving factory performance, removal of dextran is essential. The use of dextranase in sugar mills not only improves the factory performance, but also improves the sugar quality (Fulcher and Inkerman 1974). The excessive elongation of the crystal and viscosity of syrups and molasses may be reduced by the enzymatic decomposition of dextran (Hidi 1975). In the case of metabolite production by spongeassociated microorganisms, previous research has mainly focused on marine enzymes such as lipase, chitinase and alpha-amylase (Zhang et al. 2009; Han et al. 2008; Kar et al. 2009), but their production by microbial cultivation has rarely been reported (Chu et al. 2012). Besides using a 
productive strain, optimized culture medium composition and fermentation conditions play a significant role in the improvement of dextranase production. Among the techniques used in optimizing the culture medium, experimental statistical techniques such as Plackett-Burman design (PB) (Plackett and Burman 1946) and response surface methodology (RSM) have proved to be the most effective methods in optimizing the medium composition for enzyme production, eliminating the limitations of single-factor optimization process (Salihu et al. 2011). In this study, statistical optimization of medium constituents and cultural conditions was employed to enhance dextranase production. In the first step, a Plackett-Burman design was used to determine the likely effects of ten possible medium variables on dextranase production. Subsequently, the most significant factors affecting dextranase production were optimized using Box-Behnken design and response-surface analyses. To the best of our knowledge, there is not enough information concerning optimization of nutritional conditions for dextranase production by marine fungi using statistical experimental designs.

\section{Materials and methods}

Sponge, fungal strain and media

The Red Sea sponge Callyspongia spp. (Phylum Porifera, class Demospongiae, order Haplosclerida family Callyspongiidae) was collected from 1.5 to $20 \mathrm{~m}$ depth off the coast of Egyptian Red Sea. The collection sites included the protected area of Rass Mohamed, Nabq (Aqaba Gulf) and Suez Gulf (from $60 \mathrm{~km}$ south Suez to south Hurgada). In the laboratory, the specimens were washed by water and processed immediately. Coral Reef Ecology and Biology group, National Institute of Oceanography and Fisheries, Suez, Egypt, identified the collected marine samples. Host surface was treated with $70 \%$ ethanol, rinsed with sterile distilled water to remove the ethyl alcohol. Small pieces of inner tissue were rinsed with sterile sea water aseptically, then the inner tissue was cut into small cubes. A total of $3-5$ cubes of each sample were placed after removing any excess moisture on different isolation media containing penicillin benzyl sodium salt to avoid any bacterial growth. After 7 days of incubation, hyphae tips of the fungi were removed and transferred to potato dextrose agar plates (PDA) that contains the following components (g/l): potato peeled and diced into small pieces 200 , glucose 10 , agar 15 and the $\mathrm{pH} 7.5$ adjusted in $40 \%$ sea water. After 6-7 days, velvety colonies were observed.

Screening of fungi for dextranase production was carried out using blue-dextran as described by Barrett and Curtiss (1986). The isolate giving the highest ratio of zone of clearance to colony diameter was chosen for further studies. Dextranase production was carried out in the medium with contains $(\mathrm{g} / \mathrm{l})$ : dextran with average molecular weight (240.000) 1 , yeast extract 2.0, $\mathrm{K}_{2} \mathrm{HPO}_{4} 0.5$, $\mathrm{NaNO}_{3} 2.0, \mathrm{NaCl} 0.1, \mathrm{MgSO}_{4} .7 \mathrm{H}_{2} \mathrm{O} 0.5$ for optimization studies (Pleszczynska et al. 1997) at $30{ }^{\circ} \mathrm{C}$ in a rotatory shaking $(200 \mathrm{rpm})$. The enzyme dextranase was estimated in the broth medium after the removal of fungal mycelium.

Molecular identification

\section{Preparation of the fungal cultures}

Spores of the isolated marine strain were inoculated in $20 \mathrm{ml}$ of $20 \%$ potato dextrose Agar (PDA) media and incubated in $28 \pm 1{ }^{\circ} \mathrm{C}$ for 3 days. The cultures were filtered, the mats were collected and washed by distilled water prior to genomic DNA extraction. DNA was isolated from the mycelium using the DNA easy Mini Kit (Qiagen, Tokyo, Japan) according to the manufacturer's instructions. The DNA solution was used as the template for PCR. The oligonucleotide primers used for 18S rDNA partial sequence specific PCR were ITS1: 5'-TCCGTAGGTGAA CCTGCGG-3' ${ }^{\prime}$ and ITS4: 5'-TCCTCCGCTTCTTGATAT GC- $3^{\prime}$. The reaction mixture consisted of $1 \mu \mathrm{l}$ of template solution, $2.5 \mu \mathrm{l}$ of each primer ( $2 \mathrm{pmol})$, Go Taq flexi (2.5 U/ $\mu \mathrm{l}$, Promega) as taq polymerase, $5 \mu \mathrm{l}$ of dNTPs $(10 \mathrm{mM})$ and $5 \mu \mathrm{l}$ of $10 \times$ reaction buffer, sterilized distilled water was added to increase the volume to $50 \mu \mathrm{l}$. The PCR cycling conditions were one cycle of $94{ }^{\circ} \mathrm{C}$ for $3 \mathrm{~min}$, 30 cycles of $94{ }^{\circ} \mathrm{C}$ for $30 \mathrm{~s}, 50{ }^{\circ} \mathrm{C}$ for $2 \mathrm{~s}, 74{ }^{\circ} \mathrm{C}$ for $30 \mathrm{~s}$ and final extension step at $20^{\circ} \mathrm{C}$ for $3 \mathrm{~min}$. The PCR products were purified by GENECLEAN Kit (Q-BIOgene) according to the manufacture's instruction. Purified PCR products were used in sequencing reactions with the same primers in both directions using a BigDye Terminator v3.0 Cycle Sequencing Kit (Applied Biosystems). Sequencing was performed on ABI3730XLS (Applied Biosystems). After construction of the retrieved sequence, the whole sequence was used for searching of compatible sequences from database (http://blast.ncbi.nlm.nih.gov/Blast.cgi). The phylogenetic profile of our samples was constructed using multiple sequence alignment software http://blast.ncbi.nlm. nih.gov/Blast.cgi?CMD=Web\&PAGE_TYPE=BlastNews declaring the molecular identities with the closely related isolates in the database (Zheng et al. 2000).

\section{Assay of dextranase activity}

Dextranase activity was assayed by the method of Janson and Porath (1966). Reaction mixture containing $2 \mathrm{ml}$ of $2.5 \%$ dextran in acetate buffer $(0.1 \mathrm{M}, \mathrm{pH} 5.6)$ and $1 \mathrm{ml}$ of enzyme in a total volume of $3 \mathrm{ml}$ was incubated at $40{ }^{\circ} \mathrm{C}$ 
for $20 \mathrm{~min}$. Reaction was stopped by adding $3 \mathrm{ml}$ of DNS (dinitrosalicylic acid) reagent and absorbance was measured at $550 \mathrm{~nm}$ in UV/Vis spectrophotometer. One dextranase unit (U) is defined as the amount of enzyme which releases $1 \mu \mathrm{mol}$ of reducing end groups of glucose per minute.

\section{Optimization of process parameters}

\section{Identification of suitable variables using Plackett-Burman} $(P B)$ design

The Plackett-Burman was employed for screening the most significant fermentation parameters affecting dextranase enzyme production by fungal strain isolate (Sastry and Khan 1998). The variables chosen for the present study including medium components such as dextran, yeast extract, $\mathrm{K}_{2} \mathrm{HPO}_{4}, \mathrm{NaNO}_{3}, \mathrm{NaCl}$ and $\mathrm{MgSO}_{4} \cdot 7 \mathrm{H}_{2} \mathrm{O}$ also operating conditions such as incubation periods, inoculum size, medium volume and inoculum age; ten assigned variables in PB design of 12 experiments. Each independent variable was tested at two levels, high and low, which are denoted by $(+)$ and $(-)$, respectively. The experimental design with the name, symbol code, and actual level of the variables is shown in Table 1, whereas Table 2 shows the details of the design. PBD is based on the first order polynomial model:

$Y=\beta_{o}+\sum \beta_{i} X_{i}$

where $Y$ is the response (dextranase enzyme production), $\beta_{o}$ is the model intercept, $\beta_{i}$ is the linear coefficient and $X_{i}$ is the level of the independent variable. All the experiments were carried out in duplicates and the averages activity is reported as the final response in Table 2.

\section{Box-Behnken design}

From the regression analysis of the variables, the most significant factors for dextranase enzyme production were further optimized by the Box-Behnken statistical design (Box and Behnken 1960). They were further analyzed at three levels of concentration to find out the most optimal values for producing highly active dextranase enzyme. The three levels were coded as $-1,0$ and +1 representing low, middle and high concentrations, respectively, as shown in Table 3. According to the design, 15 combinations were tested (Table 4) and their observations were fitted to the following second order equation as represented in Eq. 2 as follows,

$$
\begin{aligned}
Y= & \beta_{o}+\beta_{1} X_{1}+\beta_{2} X_{2}+\beta_{3} X_{3}+\beta_{11} X_{1}^{2}+\beta_{22} X_{2}^{2} \\
& +\beta_{33} X_{3}^{2}+\beta_{12} X_{1} X_{2}+\beta_{13} X_{1} X_{3}+\beta_{23} X_{2} X_{3}
\end{aligned}
$$

\begin{tabular}{|c|c|c|c|}
\hline \multirow[t]{2}{*}{ Symbol code } & \multirow[t]{2}{*}{ Factors } & \multicolumn{2}{|c|}{ Experimental values } \\
\hline & & $\begin{array}{l}\text { Low level } \\
(-1)\end{array}$ & $\begin{array}{l}\text { High level } \\
(+1)\end{array}$ \\
\hline$X_{1}(\mathrm{~g} / \mathrm{l})$ & Dextran & 0.5 & 1.5 \\
\hline$X_{2}(\mathrm{~g} / \mathrm{l})$ & Yeast extract & 1 & 2 \\
\hline$X_{3}(\mathrm{~g} / \mathrm{l})$ & $\mathrm{K}_{2} \mathrm{HPO}_{4}$ & 0.5 & 1 \\
\hline$X_{4}(\mathrm{~g} / \mathrm{l})$ & $\mathrm{NaNO}_{3}$ & 1 & 2 \\
\hline$X_{5}(\mathrm{~g} / \mathrm{l})$ & $\mathrm{NaCl}$ & 0.5 & 1 \\
\hline$X_{6}(\mathrm{~g} / \mathrm{l})$ & $\mathrm{MgSO}_{4} \cdot 7 \mathrm{H}_{2} \mathrm{O}$ & 0.5 & 1 \\
\hline$X_{7}$ (days) & Incubation time & 5 & 7 \\
\hline$X_{8}(\mathrm{ml})$ & Inoculum size & 1 & 2 \\
\hline$X_{9}(\mathrm{ml} / 250 \mathrm{ml}$ flask $)$ & Medium volume & 25 & 50 \\
\hline$X_{10}(\mathrm{~h})$ & Inoculum age & 48 & 72 \\
\hline
\end{tabular}

Table 1 Experimental definition for the Plackett-Burman design

Table 2 Two-level factorial design of variables (in coded levels) with titer as response values

\begin{tabular}{lrrrrrrrrrrl}
\hline Run & $X_{1}$ & $X_{2}$ & $X_{3}$ & $X_{4}$ & $X_{5}$ & $X_{6}$ & $X_{7}$ & $X_{8}$ & $X_{9}$ & $X_{10}$ & $\begin{array}{l}\text { Dextranase } \\
\text { activity (U/ml) }\end{array}$ \\
\hline 1 & 1 & -1 & 1 & 1 & 1 & -1 & 1 & -1 & -1 & -1 & 358.5 \\
2 & 1 & -1 & -1 & 1 & 1 & 1 & -1 & 1 & 1 & -1 & 327.0 \\
3 & -1 & -1 & -1 & -1 & 1 & 1 & 1 & -1 & 1 & 1 & 351.0 \\
4 & 1 & 1 & 1 & -1 & -1 & 1 & 1 & 1 & -1 & -1 & 321.9 \\
5 & 1 & -1 & 1 & -1 & -1 & -1 & -1 & 1 & 1 & 1 & 420.6 \\
6 & 1 & 1 & -1 & 1 & -1 & -1 & -1 & -1 & 1 & 1 & 499.5 \\
7 & -1 & 1 & 1 & -1 & 1 & -1 & 1 & 1 & 1 & -1 & 265.2 \\
8 & -1 & -1 & 1 & 1 & -1 & 1 & 1 & 1 & -1 & 1 & 274.8 \\
9 & -1 & 1 & -1 & 1 & 1 & -1 & -1 & 1 & -1 & 1 & 212.1 \\
10 & 1 & 1 & 1 & -1 & 1 & 1 & -1 & -1 & -1 & 1 & 333.9 \\
11 & -1 & 1 & 1 & 1 & -1 & 1 & -1 & -1 & 1 & -1 & 349.5 \\
12 & -1 & -1 & -1 & -1 & -1 & -1 & -1 & -1 & -1 & -1 & 321.9 \\
\hline
\end{tabular}

Variables coded are same as given in Table 1

where $Y$ is the measured response (dextranase enzyme production), $X_{1}, X_{2}$ and $X_{3}$ are independent variables, which influence the response variable $Y, \beta_{1}, \beta_{2}, \beta_{3}$ are linear coefficients, $\beta_{12}, \beta_{22}$ and $\beta_{33}$ are cross-product coefficients and $\beta_{11}, \beta_{22}$ and $\beta_{33}$ are quadratic coefficients. The quality of the fit of the polynomial model equation is expressed by the coefficient of determination $R^{2}$.

\section{Data analysis and optimization}

The data of the enzyme activity of each trial was subjected to analysis, using statistical tool Minitab 16 software for Plackett-Burman and the Box-Behnken experiment. Statistical analysis of the model was performed 
Table 3 Coded and actual values of the culture conditions tested in Box-Behnken design

\begin{tabular}{lllcc}
\hline Symbol code & Factors & \multicolumn{3}{l}{$\begin{array}{l}\text { Actual levels of coded } \\
\text { factors }\end{array}$} \\
\cline { 3 - 5 } & & +1 & 0 & -1 \\
\hline$X_{1}(\mathrm{~g} / \mathrm{l})$ & Dextran & 2.5 & 2.0 & 1.5 \\
$X_{9}(\mathrm{ml} / 250 \mathrm{ml}$ flask $)$ & Medium volume & 65 & 50 & 25 \\
$X_{10}(\mathrm{~h})$ & Inoculum age & 96 & 72 & 48 \\
\hline
\end{tabular}

Table 4 Box-Behnken of three variables in coded along with titer as response values

\begin{tabular}{lrrrl}
\hline Run & $X_{1}$ & $X_{9}$ & $X_{10}$ & $\begin{array}{l}\text { Dextranase } \\
\text { activity }(\mathrm{U} / \mathrm{ml})\end{array}$ \\
\hline 1 & -1 & -1 & 0 & 326.70 \\
2 & 1 & -1 & 0 & 348.06 \\
3 & -1 & 1 & 0 & 357.70 \\
4 & 1 & 1 & 0 & 414.90 \\
5 & -1 & 0 & -1 & 271.80 \\
6 & 1 & 0 & -1 & 362.40 \\
7 & -1 & 0 & 1 & 393.60 \\
8 & 1 & 0 & 1 & 398.40 \\
9 & 0 & -1 & -1 & 257.70 \\
10 & 0 & 1 & -1 & 403.20 \\
11 & 0 & -1 & 1 & 395.66 \\
12 & 0 & 1 & 1 & 403.20 \\
13 & 0 & 0 & 0 & 429.00 \\
14 & 0 & 0 & 0 & 429.00 \\
15 & 0 & 0 & 0 & 429.00 \\
\hline
\end{tabular}

through the analysis of variance (ANOVA) to evaluate the statistical significance of the model. The models of each response were expressed in terms of uncoded variables. The quality of the polynomial model equation was judged statistically by the coefficient of determination $R^{2}$, adjusted $R^{2}$, and predicted $R^{2}$. The determination coefficient $\left(R^{2}\right)$ is a measure of how well the regression equation fits the sample data. Adjusted $R^{2}$ is a modification of $R^{2}$ that adjusts for the number of explanatory terms in a model. A predicted $R^{2}$ is used to measure the amount of variation in new data (i.e., other levels among the tested maximal value and minimal value of the factor) explained by the model. Statistical significance was determined by an $F$ test at $5 \%$ level. The significance of the regression coefficients was tested by a $t$ test. STATISTICA sigma software (Version 8.0, StatSoft Inc., USA) was used to plot Pareto chart of standardized effects, the three-dimensional surface plots and contour plots, in order to illustrate the relationship between the response and the experimental levels of each of the variables utilized in this study.

\section{Results and discussion}

Identification of active marine endophyte

The aim of this study was to find strains of marine fungi for the production of dextranase with potential industrial applications. The identification of fungi is mainly done by recognizing the morphological features of genera and species by macroscopic and microscopic examination. Among molecular techniques, PCR-specific amplification is a rapid method used in the direct detection of DNAs and RNAs of microorganisms from clinical and environmental samples to accurately and quantitatively ascribe microorganism compositions (Zhihong et al. 2003). The fungal strain isolated from Red Sea sponge Callyspongia spp. exhibited dextranase activity on dextran medium. It gave a zone of clearance to colony diameter ratio of $2.0 \mathrm{~cm}$ which was higher than the clearance displayed by other strains obtained from the region and was chosen for molecular identification technique. According to sequencing similarities and multiple alignment, the fungus was found to be in a close relation to Aspergillus flocculosus strain NRRL 5224 (ac: EU021616.1) with a $99 \%$ identity (Table 5); accordingly, the strain is hereafter referred to as Aspergillus flocculosus EU NRC. Since the organism appeared promising, experiments were devised to optimize the cultural conditions. It was reported before that, fungi and bacteria were identified as the main enzymatic sources capable of

Table 5 Distribution of the first ten blast hits on the query sequence

\begin{tabular}{|c|c|c|c|c|}
\hline \multirow[t]{2}{*}{ Organisms description } & \multirow{2}{*}{$\begin{array}{l}\text { GenBank } \\
\text { accession }\end{array}$} & \multicolumn{3}{|c|}{ Identities } \\
\hline & & Match & Total & $\begin{array}{l}\text { Pct. } \\
(\%)\end{array}$ \\
\hline $\begin{array}{l}\text { Aspergillus flocculosus } \\
\text { strain NRRL } 5224\end{array}$ & EU021616.1 & 587 & 589 & 99 \\
\hline Aspergillus sp. r089 & HQ649845.1 & 582 & 587 & 99 \\
\hline $\begin{array}{l}\text { Aspergillus } \\
\text { ochraceopetaliformis } \\
\text { strain RKI08-134 }\end{array}$ & FJ797698.1 & 583 & 589 & 99 \\
\hline $\begin{array}{l}\text { Aspergillus } \\
\text { ochraceopetaliformis } \\
\text { isolate NRRL } 35668\end{array}$ & EF661432.1 & 575 & 577 & 99 \\
\hline $\begin{array}{l}\text { Aspergillus } \\
\text { ochraceopetaliformis } \\
\text { isolate NRRL } 35055\end{array}$ & EF661431.1 & 575 & 577 & 99 \\
\hline $\begin{array}{l}\text { Aspergillus } \\
\text { ochraceopetaliformis } \\
\text { isolate NRRL } 4752\end{array}$ & EF661429.1 & 575 & 577 & 99 \\
\hline Aspergillus sp. F5 & FJ214372.1 & 581 & 588 & 99 \\
\hline Aspergillus sp. r192 & HQ649847.1 & 576 & 581 & 99 \\
\hline Aspergillus sp. OY10607 & FJ571434.1 & 567 & 568 & 99 \\
\hline $\begin{array}{l}\text { Aspergillus } \\
\text { ochraceopetaliformis } \\
\text { strain SCSGAF0071 }\end{array}$ & JN851013.1 & 563 & 568 & 99 \\
\hline
\end{tabular}


hydrolyzing dextrans. In the early 1950s Japanese researchers identified Penicillium lilacinum and Penicillium funiculosum fungi that produced dextranase in the presence of dextrans and later in the 1960s others strains from Chaetomium gracile and Gibellela funiculosum fungi. After an extensive search that continued in Japan during the 1970s, the Aspergillus carneus fungus strain that accumulates the enzyme when cultured in dextrans and another from the Penicillium luteum were identified (Novo 1977). The dextranase from $P$. lilacinum showed maximum activity in the $\mathrm{pH}$ range of 5.0-5.5 and between 53 and $60{ }^{\circ} \mathrm{C}$ (Fukumoto et al. 1971). Similar observations were reported for the production of dextranase by various fungi (Hattori and Ishibashi 1981; Shukla and Madhu 1989; Madhu 1984).

Screening of important variables for dextranase production using Plackett-Burman design

The influence of dextran, yeast extract, $\mathrm{K}_{2} \mathrm{HPO}_{4}, \mathrm{NaNO}_{3}$, $\mathrm{NaCl}, \mathrm{MgSO}_{4} \cdot 7 \mathrm{H}_{2} \mathrm{O}$ concentrations as well as incubation time, inoculum size, medium volume and inoculum age on dextranase enzyme production were investigated with the help of PBD. According to Davis et al. (1974) corn steep liquor and autolyzed yeast extract were commonly used for the dextranase enzyme production. The data in Table 2 indicated that there was a wide variation from 212 to $499.5 \mathrm{U} / \mathrm{ml}$ of dextranase enzyme in the 12 runs which reflects the variations caused due to the presence of different factors influencing the activity at low and high levels considered in various combinations.

Regression analysis and the analysis of variance (ANOVA) showed probability values $<0.05(p<0.05)$ indicated significance of the model term, whereas $p$ values $>0.1(p>0.1)$ indicated model terms were not significant as shown in Tables 6 and 7. The $p$ value is the probability that the magnitude of a contrast coefficient is due to random process variability and serves as a tool for checking the significance of each of the coefficients, low $p$ value indicates a real or significant effect (Levine 2005). On the same basis, dextran $(t=55.97, p=0.011)$, medium volume $(t=44.79$, $p=0.014)$ and inoculum age $(t=17, p=0.037)$ with higher $t$ value and lower $p$ values were considered as the most significant components (Table 6). When the sign of the concentration effect of the tested variable is positive, the influence of the variable upon dextranase enzyme production is greater at a high concentration and when negative, the influence of the variable is greater at a low concentration. The effect of variables $X_{2}$ (yeast extract), $X_{5}(\mathrm{NaCl}), X_{6}$ $\left(\mathrm{MgSO}_{4} \cdot 7 \mathrm{H}_{2} \mathrm{O}\right)$ and $X_{8}$ (inoculum size) are $-23.9,-227$, -79.8 and -130.9 , respectively, i.e., the influence of these four variables is greater at a low concentration. The goodness of the fit of the model can be checked by the 'determination coefficient' $R^{2}$, the value of $R^{2}$ and adjusted $R^{2}$ are 99.9 and $99.8 \%$, respectively, which shows a high correlation between the observed values and the predicted values. This means that regression model provides an excellent explanation of the relationship between the independent variables (factors) and the response (dextranase enzyme production). On application of ANOVA, it was found that the first order model for dextranase enzyme production was fitted to the results obtained from the 12 experiments as the equation:

$$
\begin{aligned}
Y= & 280+81.15 X_{1}-11.95 X_{2}-10.3 X_{3}+1.15 X_{4} \\
& -113 X_{5}-39.9 X_{6}+8.83 X_{7}-65.5 X_{8}+2.60 X_{9} \\
& +1.03 X_{10}
\end{aligned}
$$

where, $Y$ predicted response and $X_{1}, X_{2}, X_{3}, X_{4}, X_{5}, X_{6}, X_{7}$, $X_{8}, X_{9}$ and $X_{10}$ are the coded values of dextran, yeast extract, $\mathrm{K}_{2} \mathrm{HPO}_{4}, \mathrm{NaNO}_{3}, \mathrm{NaCl}, \mathrm{MgSO}_{4} \cdot 7 \mathrm{H}_{2} \mathrm{O}$, incubation time, inoculum size, medium volume and inoculum age, respectively.

The main effect is present when different levels of a factor affect the characteristic differently. Minitab statisti-

\begin{tabular}{|c|c|c|c|c|c|c|}
\hline Code & Variable & Effect & $t$ test & $F$ ratio & $B$ coefficient & $p$ value \\
\hline$X_{1}$ & Dextran $(g / l)$ & 162.3 & 55.97 & $3,132.14$ & 81.1500 & $0.011^{*}$ \\
\hline$X_{2}$ & Yeast extract $(\mathrm{g} / \mathrm{l})$ & -23.9 & -8.24 & 67.92 & -11.9500 & 0.077 \\
\hline$X_{3}$ & $\mathrm{~K}_{2} \mathrm{HPO}_{4}(\mathrm{~g} / \mathrm{l})$ & -10.300 & -3.55 & 12.61 & -10.3 & 0.175 \\
\hline$X_{4}$ & $\mathrm{NaNO}_{3}(\mathrm{~g} / \mathrm{l})$ & 2.3 & 0.79 & 0.63 & 1.15000 & 0.573 \\
\hline$X_{5}$ & $\mathrm{NaCl}(\mathrm{g} / \mathrm{l})$ & -227.0 & -39.14 & $1,531.78$ & -113.500 & $0.016^{*}$ \\
\hline$X_{6}$ & $\mathrm{MgSO}_{4} \cdot 7 \mathrm{H}_{2} \mathrm{O}(\mathrm{g} / \mathrm{l})$ & -79.8 & -13.76 & 189.30 & -39.9 & 0.046 \\
\hline$X_{7}$ & Incubation time (days) & 17.6 & 12.17 & 148.17 & 8.82500 & 0.052 \\
\hline$X_{8}$ & Inoculum size $(\mathrm{ml})$ & -130.9 & -45.14 & $2,037.43$ & -65.4500 & $0.014^{*}$ \\
\hline$X_{9}$ & Medium volume (ml/250 mlflask) & 5.2 & 44.79 & $2,006.42$ & 2.59800 & $0.014^{*}$ \\
\hline$X_{10}$ & Inoculum age $(\mathrm{h})$ & 2.1 & 17.00 & 289.00 & 1.02708 & $0.037 *$ \\
\hline
\end{tabular}
cal software generates the main effect plot by plotting the

Table 6 Estimated regression coefficients for dextranase enzyme production

* Identifies variables with a significant effect on the response $(p$ value $<0.05$ ) 


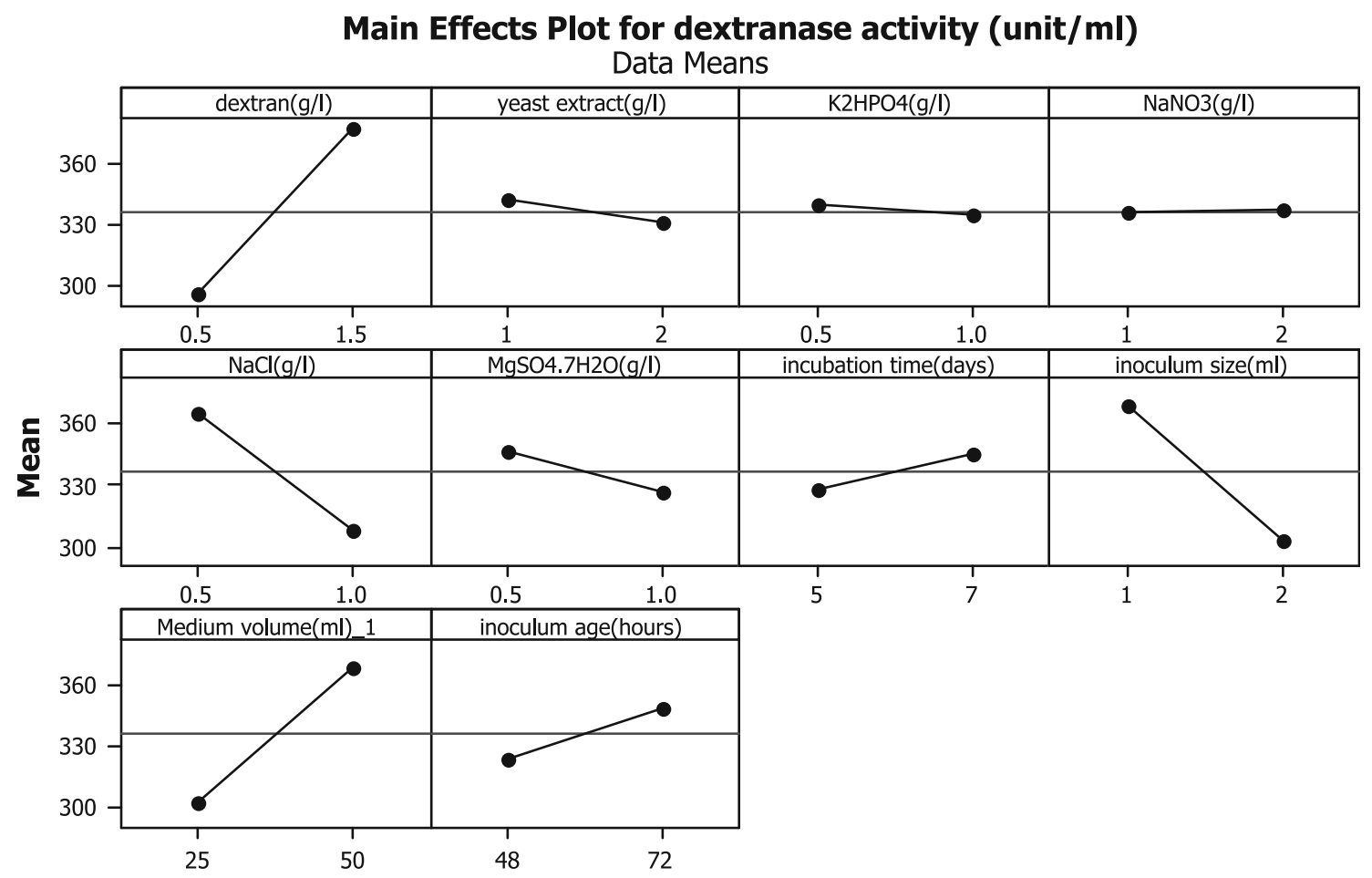

Fig. 1 Figure shows the main effect plots for the system, which show how each factor affects the response characteristic

Table 7 Analysis of variance (ANOVA) for the quadratic model

\begin{tabular}{lrrrrl}
\hline Source & $d f$ & SS & MS & $F$ & $p$ \\
\hline Regression & 10 & $59,387.7$ & $5,938.8$ & 941.54 & 0.025 \\
Residual error & 1 & 6.3 & 6.3 & & \\
\hline$R^{2}=99.99 \%, R^{2}(\mathrm{pred})=98.47 \%, R^{2}(\mathrm{adj})=99.88 \%$ & \\
$S S$ sum of squares, $d f$ degrees of freedom, $M S$ mean square
\end{tabular}

characteristic average for each factor level (Fig. 1). These averages are the same as those shown in Table 6. A line connects the points for each factor. When the line is horizontal (parallel to the $x$-axis), main effect is not present. Different levels of the factor affect the characteristic differently. The greater the difference in the vertical position of the plotted points (the more the line is not parallel to the $x$-axis), the greater the magnitude of the main effect. Analysis of the measured response variables enabled to obtain standardized Pareto charts and response surface plots. A standardized Pareto chart consists of bars with a length proportional to the absolute value of the estimated effects, divided by the standard error. The bars are displayed in order of the size of the effects, with the largest effects on top. A high $t$ test value and a low probability indicated a high significance (Niladevi et al. 2009). The chart includes a vertical line at the critical $t$ value for an alpha of 0.05. Bars are displayed in order of the size of the effects, and the standardized effect of each term was shown on the top of its corresponding bar (Fig. 2).
Results showed that, variations of dextranase production in the 15 trials ranged from 257.70 to $429 \mathrm{U} / \mathrm{ml}$ (Table 4).

Regression analysis in Table 8 of the experimental data shows that dextran, medium volume and inoculum age had positive linear effects on enzyme synthesis $(p<0.05)$. Probability $(p)$ values were used as a tool to check the significance of each of the coefficients. The smaller the magnitude of $p$ value, the more significant was the correlation with the corresponding coefficient (Sreekumar and Krishnan 2010). Among the three factors tested, inoculum age had the highest impact on dextranase enzyme activity as given by the highest linear coefficient (732.90), followed by inoculum age (17.03) and medium volume (9.83). These factors also showed significant negative quadratic effects on enzyme production indicating that dextranase enzyme activity increased as the level of these factors decreased and decreased as the level of these parameters increased above certain values. Interaction between these parameters was also significant. The interactions between dextran concentration-inoculum age and medium volume-inoculum age were significant as shown by low $p$ values $(p<0.05)$ for interactive terms. But the interaction between dextran concentration-medium volume was found to be insignificant as given by $p$ value above 0.05 . Hence this term was excluded from the quadratic polynomial Eq. 2 used for this model. 
Fig. 2 Pareto chart of standardized effects on the dextranase enzyme production. The above results indicated that the Plackett-Burman design is a powerful tool for identifying factors, which had significant influence on dextranase activity. The exact optimal values of the most significant factors (dextran, medium volume and inoculum age) can be determined by the subsequent Box-Behnken experiment. Optimization of significant culture parameters with RSM based on the Box-Behnken design. The culture variables found to significantly affect dextranase enzyme production were tested at values given in Table 6 for the Box-Behnken design to optimize the magnitude of those variables
Pareto Chart of Standardized Effects; Variable: Dextranase activity (unit/ml) 10 factors at two levels; MS Residual=6.3075

DV: Dextranase activity (unit/ml)

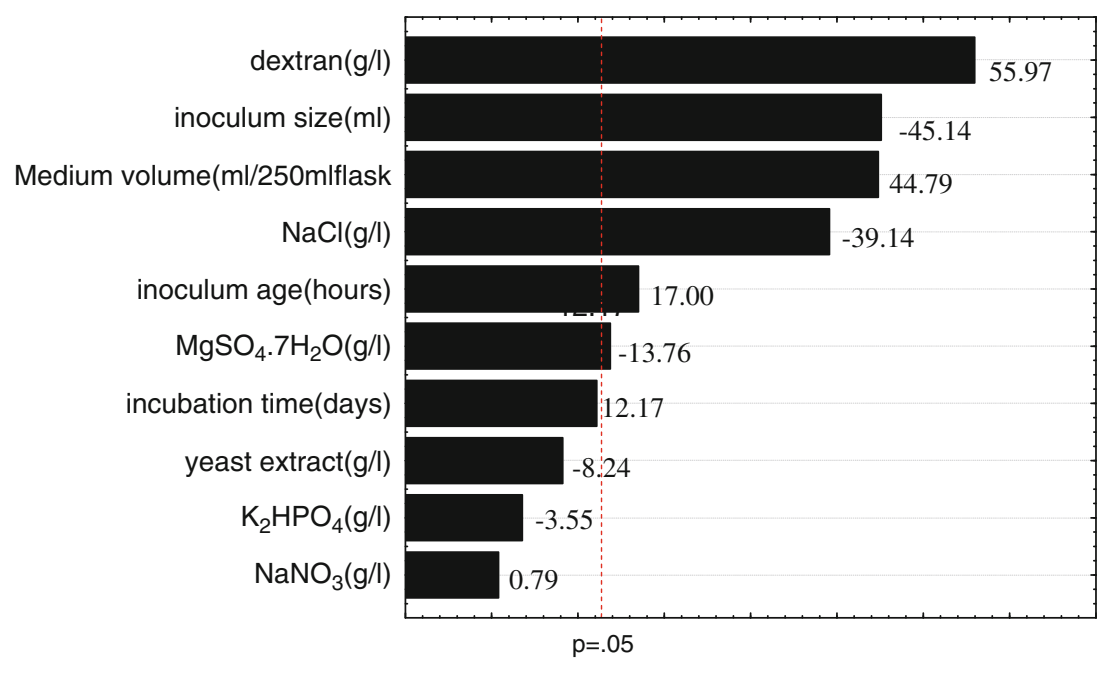

Standardized Effect Estimate (Absolute Value)

Table 8 Estimated regression coefficients of Box-Behnken design for dextranase enzyme production

\begin{tabular}{|c|c|c|c|c|c|}
\hline Term & & Coefficients & SE coefficients & $t$ value & $p$ value \\
\hline Constant & & $-1,282.69$ & 161.252 & -7.955 & $0.001^{*}$ \\
\hline Dextran & $X_{1}$ & 732.90 & 112.166 & 6.534 & $0.001^{*}$ \\
\hline Medium volume & $X_{9}$ & 9.83 & 2.143 & 4.586 & $0.006^{*}$ \\
\hline Inoculum age & $X_{10}$ & 17.03 & 1.987 & 8.572 & $0.000^{*}$ \\
\hline Dextran $\times$ dextran & $X_{1} \times X_{1}$ & -151.10 & 25.430 & -5.942 & $0.002 *$ \\
\hline Medium volume $\times$ medium volume & $X_{9} \times X_{9}$ & -0.06 & 0.017 & -3.341 & 0.021 \\
\hline Inoculum age $\times$ inoculum age & $X_{10} \times X_{10}$ & -0.06 & 0.011 & -5.454 & $0.003^{*}$ \\
\hline Dextran $\times$ medium volume & $X_{1} \times X_{9}$ & 0.92 & 0.601 & 1.529 & 0.187 \\
\hline Dextran $\times$ inoculum age & $X_{1} \times X_{10}$ & -1.79 & 0.509 & -3.512 & $0.017 *$ \\
\hline Medium volume $\times$ inoculum age & $X_{9} \times X_{10}$ & -0.07 & 0.013 & -5.460 & $0.003^{*}$ \\
\hline
\end{tabular}

$R^{2}=98.19 \%, R^{2}$ (pred) $=68.97 \%, R^{2}(\operatorname{adj})=94.93 \%$

$S E$ standard error, $t$ Student's test, $p$ corresponding level of significance

* Significant

$$
\begin{aligned}
Y= & -1,282.69+732.90 X_{1}+9.83 X_{9}+17.03 X_{10} \\
& -151.10 X_{1} X_{1}-0.06 X_{9} X_{9}-0.06 X_{10} X_{10} \\
& -1.79 X_{1} X_{10}-0.07 X_{9} X_{10}
\end{aligned}
$$

where $Y$ is the response (dextranase enzyme activity) and $X_{1}, X_{9}$ and $X_{10}$, are the coded values of the independent variables.

The adequacy of the model was checked using analysis of variance (ANOVA) and the results were presented in Table 9. The correlation coefficient $\left(R^{2}=98.19 \%\right.$ and adjusted coefficient $R^{2}$ (adjusted) $=94.93 \%$ were also high, which indicates a high significance of the experiments (Akhnazarova and Kafarov 1982).

The ANOVA of the regression model demonstrates that the model is highly significant. This is evident from the calculated $F$ value (Thompson et al. 1994; Khuri and Cornell 1987) where $(F$ model $=30.10)$ and probability value $(p=0.001)$. It is evident that the linear $(p=0.001)$ quadratic effect $(p=0.003)$ and interaction effect $(p=0.006)$ of the variables had greater influence on dextranase enzyme production. The model determination coefficient $R^{2}$ was reasonable and in agreement with the experimental results, indicating that $98.87 \%$ of the variability could be revealed by the model (Table 9). Accordingly, three-dimensional graphs were generated for the pair-wise combination of the three factors, while keeping the third one at its middle point levels. From the middle point of the bump of the $3 \mathrm{D}$ plot, the optimal conditions of the production medium was identified. 
Table 9 ANOVA for response surface quadratic model

\begin{tabular}{lcccccc}
\hline Source & Degrees of freedom & Sum of square & Adjusted sum of square & Mean of square & $F$ value & $p$ value \\
\hline Regression & 9 & $40,429.4$ & $40,429.4$ & $4,492.2$ & 30.10 & 0.001 \\
Linear & 3 & $23,800.7$ & $15,210.1$ & $5,070.0$ & 33.97 & 0.001 \\
Square & 3 & $9,989.6$ & $9,989.6$ & $3,329.9$ & 22.31 & 0.003 \\
Interaction & 3 & $6,639.1$ & $6,639.1$ & $2,213.0$ & 14.83 \\
Residual error & 5 & 746.2 & 746.2 & 249.2 & 0.006 \\
Lack-of-fit & 3 & 746.2 & 746.2 & 0.0 & \\
Pure error & 2 & 0.0 & 0.0 & & \\
Total & 14 & $41,175.6$ & & &
\end{tabular}

$R^{2}=98.87 \%, R^{2}$ (pred) $=81.91 \%, R^{2}(\operatorname{adj})=96.83 \%$

$F$ Fishers's function, $p$ corresponding level of significance

Fig. 3 a Response surface and b contour plots for the interaction of dextran concentration and medium volume at inoculum age $72 \mathrm{~h}$ on dextranase enzyme activity. The values in the figure indicated the level of dextranase enzyme activity $(\mathrm{U} / \mathrm{ml})$ (a) 3D Surface Plot of Dextranase activity (unit/ml) against dextran(g/l) and Medium volume ( $\mathrm{ml} / 250 \mathrm{mlflask})$

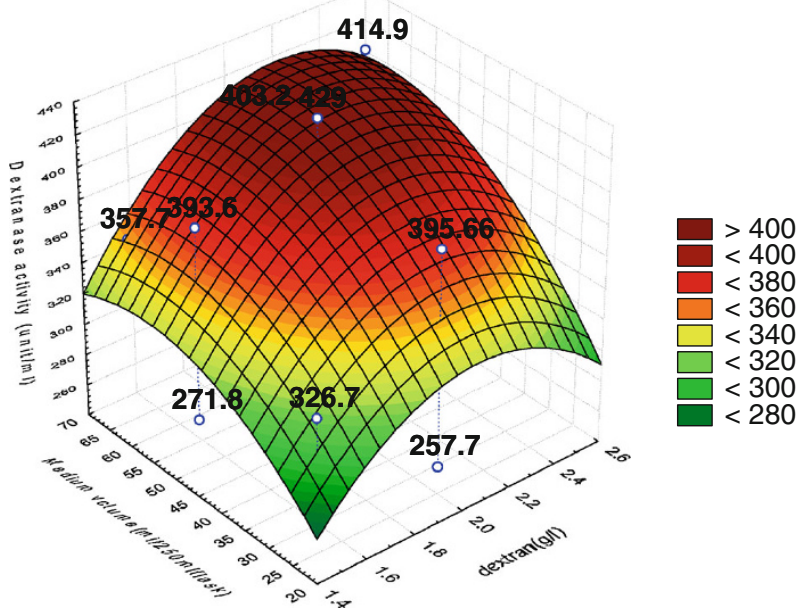

(b) 3D Contour Plot of Dextranase activity (unit/ml) against dextran(g/l) and Medium volume( $\mathrm{ml} / 250 \mathrm{mlflask})$

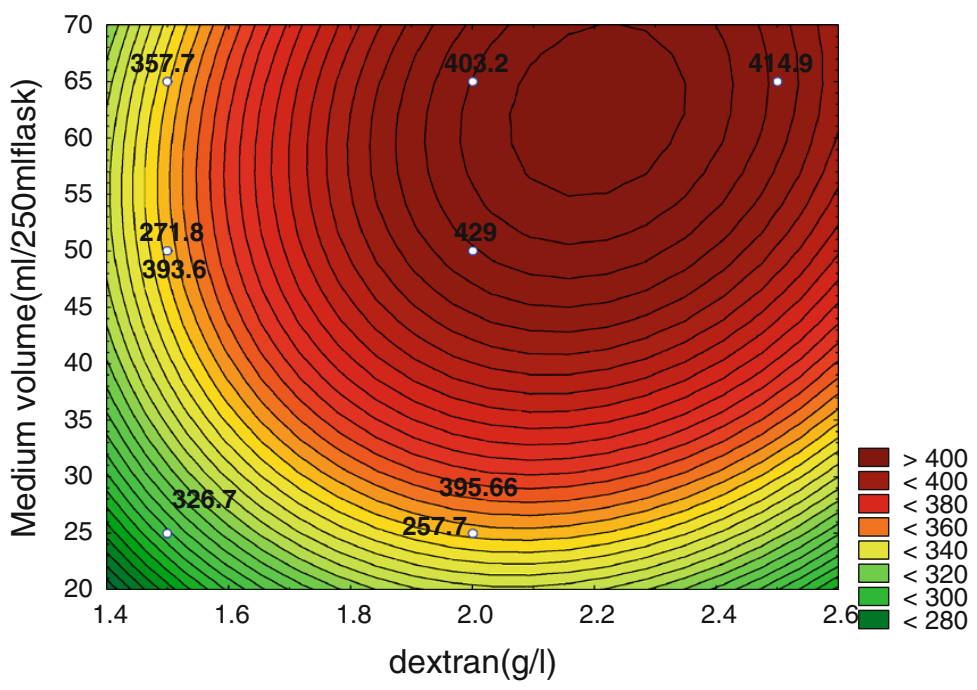


Fig. 4 a Response surface and b contour plots for the interaction of dextran concentration and inoculum age at medium volume $50 / 250 \mathrm{ml}$ flask on dextranase enzyme activity. The values in the figure indicated the level of dextranase enzyme activity $(\mathrm{U} / \mathrm{ml})$ (a) 3D Surface Plot of Dextranase activity (unit/ml) against dextran(g/l) and inoculum age(hours)

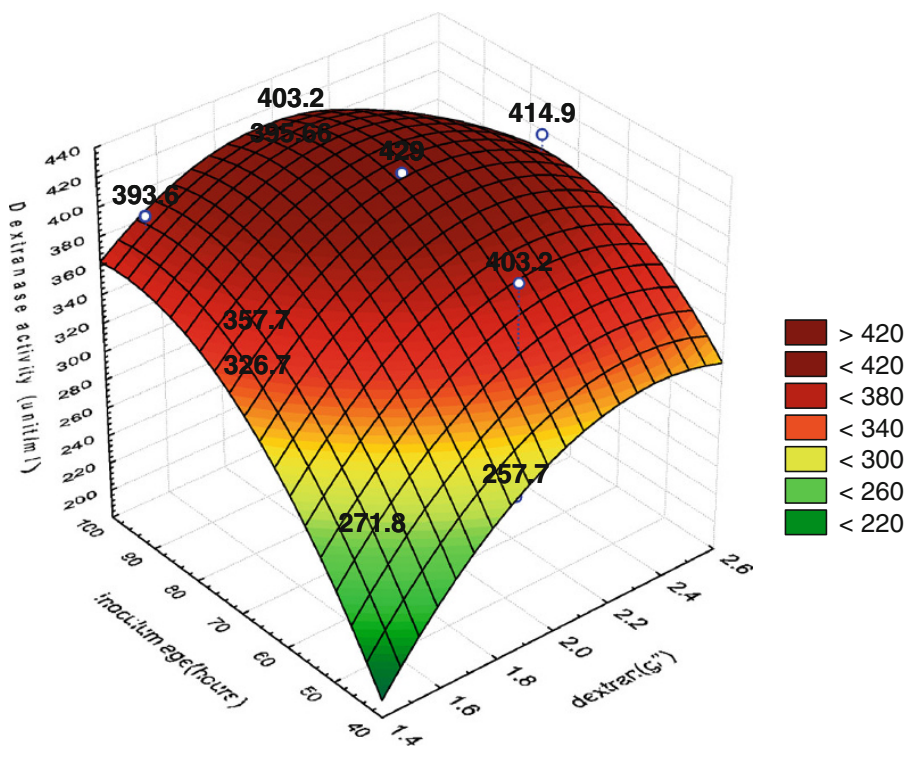

(b) 3D Contour Plot of Dextranase activity (unit/ml) against dextran(g/l) and inoculum age(hours)

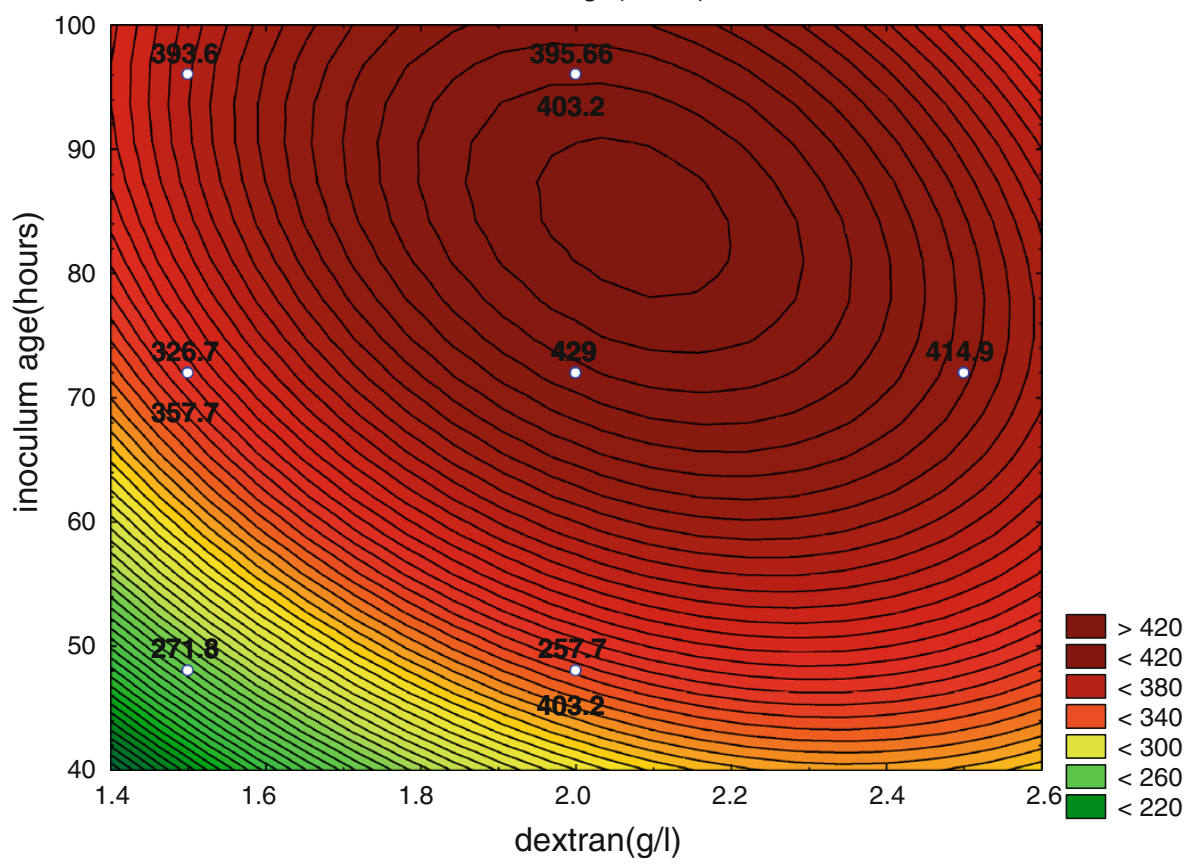

Figure 3a represent three-dimensional curve of the calculated response surface from the interaction effect of dextran concentration and medium volume on dextranase enzyme production, whereas inoculum age was kept at its middle level, that is, $72 \mathrm{~h}$. The response surface plot indicated that dextranase enzyme production increased with the increase in both dextran concentration and medium volume. With further increase in dextran concentration and medium volume, the yield slightly decreased this is may be due to excess accumulation of dextran in medium which increases the viscosity and ultimately hinders the fungal growth, resulting in decreased production of dextranase.

Figure 4a shows the interaction effect of dextran concentration and inoculum age on dextranase enzyme production, whereas medium volume was kept at its middle level, 50/250 ml flask. The dextranase enzyme production increased with the increase of medium volume with further increase in inoculum age, the yield slightly decreased 
Fig. 5 a Response surface and b contour plots for the interaction of medium volume $(\mathrm{ml} / 250 \mathrm{ml}$ flask) and inoculum age (h) at dextran concentration $2 \mathrm{~g} / \mathrm{l}$ on dextranase enzyme activity. The values in the figure indicated the level of dextranase enzyme activity $(\mathrm{U} / \mathrm{ml})$ (a) 3D Surface Plot of Dextranase activity (unit/ml) against Medium volume( $\mathrm{ml} / 250 \mathrm{~m} / \mathrm{flask}$ ) and inoculum age(hours)

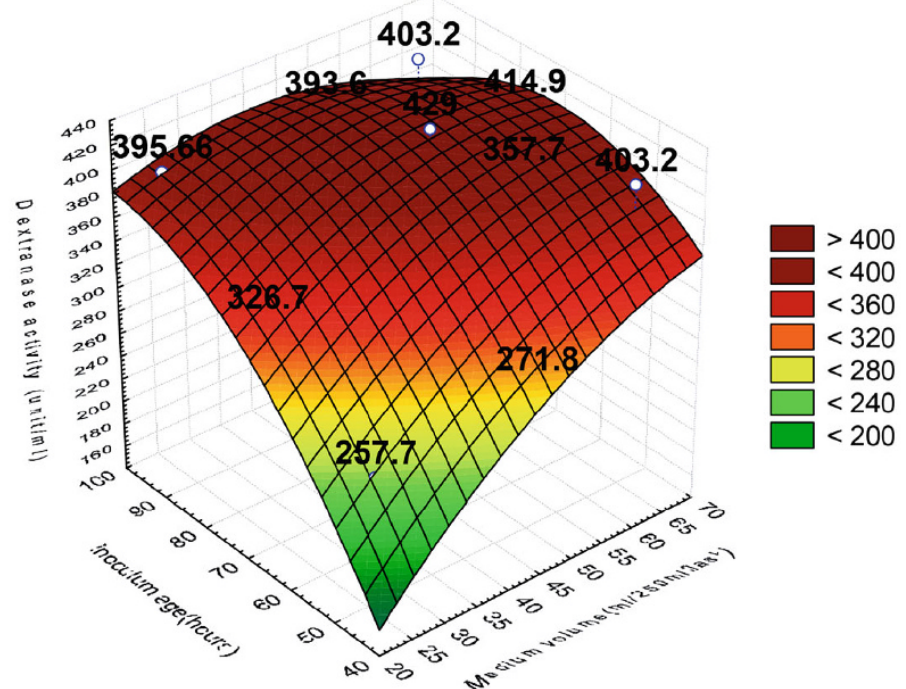

(b) 3D Contour Plot of Dextranase activity (unit/ml) against Medium volume(ml/250mlflask) and inoculum age(hours)

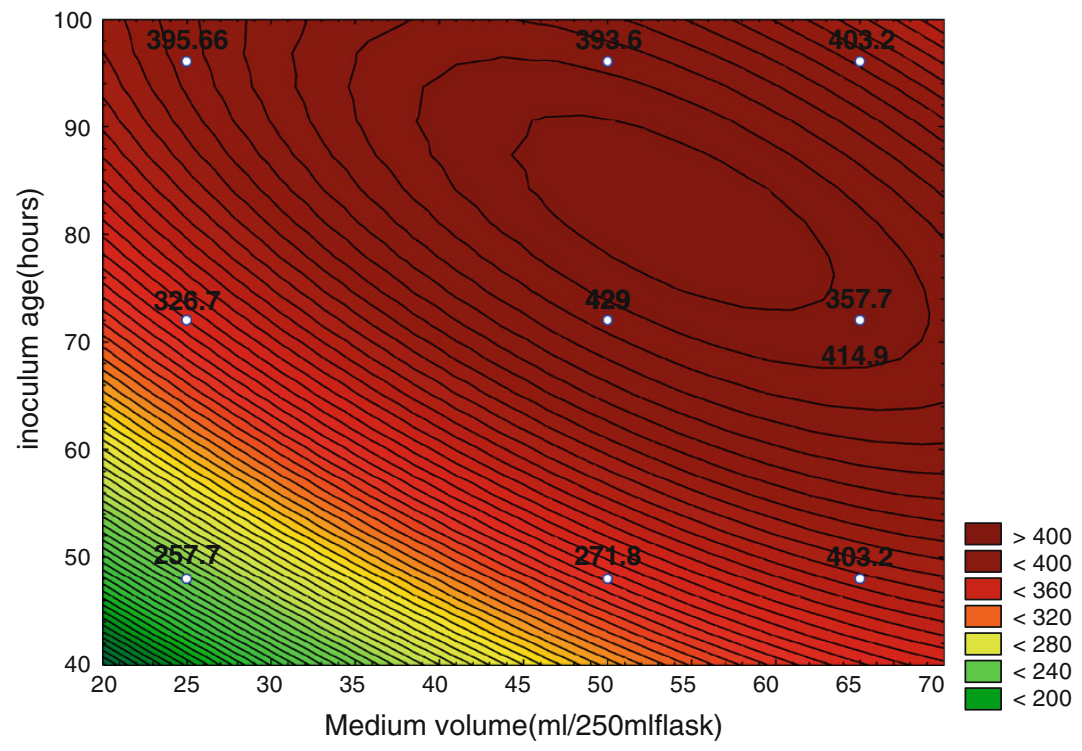

which may be due to the occurrence of microbial death phase.

Figure 5a represents the interactive effect of medium volume and inoculum age on dextranase enzyme production, whereas dextran concentration was kept at its middle level, $2 \mathrm{~g} / \mathrm{l}$. The production increased with the increase of medium volume and inoculum age, the yield slightly decreased with further increase in medium volume and inoculum age which may be due to the occurrence of microbial death phase.

The yield values for different concentrations of the variables can also be predicted from the respective contour plots (Box et al. 1978; Box and Wilson 1951; Khuri and
Cornell 1987). The maximum predicted yield is indicated by the surface confined in the smallest ellipse in the contour diagram. The contour plots for each of the responses generated are shown in Figs. 3, 4, 5b. All the contour plots are elliptical indicating that there is a perfect interaction between the independent variables (Muralidhar et al. 2001). Higher production was recorded in Fig. 3b when dextran concentration is in the range of $2.1-2.3 \mathrm{~g} / \mathrm{l}$ and medium volume is in the range of 52-70 ( $\mathrm{ml} / 250 \mathrm{ml}$ flask). Similar pattern of highest enzymatic production was also recoded from Fig. $4 \mathrm{~b}$ when dextran concentration is in the range of 2-2.2 $\mathrm{g} / \mathrm{l}$ and the inoculum age is in the range of 80-90 h, whereas Fig. 5b shows medium volume of 45-65 
Fig. 6 Recommended input variables to achieve optimal response

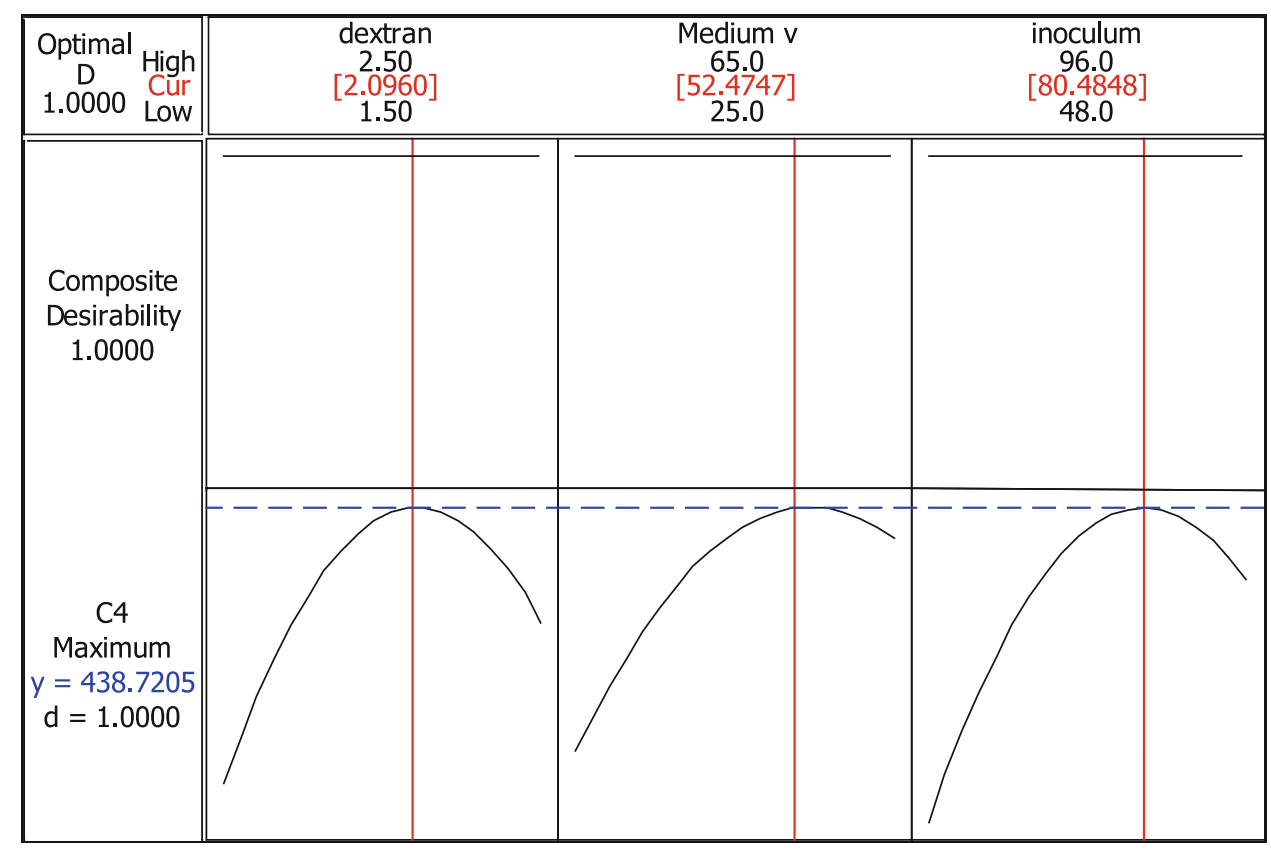

$(\mathrm{ml} / 250 \mathrm{ml}$ flask) and inoculum age is in the range of 70-90 h.

Eventually, after making the regression model, a numerical optimization method by desirability function was implied to optimize the response. The graph in Fig. 6 indicates how individual factors in each column influence the response while the other factors are held constant. The values between high and low values optimal parametric setting were recommended by the minitab 16 software to obtain the most suitable responses. $D$ is the composite desirability and $d$ is the individual desirability. The maximum values for $D$ and $d$ are 1.0000 (Myers and Montgomery 1995). Figure 6 shows values for $D$ and $d$ in optimal conditions as 1.0000 , confirming that the model proposed is suitable.

In the recommended optimal model (see Fig. 6), parametric settings of dextran concentration of $2.09 \mathrm{~g} / \mathrm{l}$, medium volume of $52.5 / 250 \mathrm{ml}$ flask and inoculum age of $80.5 \mathrm{~h}$, were set. The response for this set of values for dextranase enzyme production with desirability of 1.0000 was $438.72 \mathrm{U} / \mathrm{ml}$.

Therefore, the predicted optimum condition was verified experimentally and compared with the predicted data. The measured dextranase activity was $440 \mathrm{U} / \mathrm{ml}$ higher than many fungi which synthesizes dextranase including Penicillium funiculosum, P. notatum, Fusarium moniliforme, Aspergillus carneus (Abdel-Naby et al. 1999; Fukumoto et al. 1971; Pleszczynska et al. 1997; Hiraoka et al. 1972; Simonson et al. 1975). The verification revealed a high degree of accuracy of the model of more than $96.7 \%$, indicating the model validation under the tested conditions. Therefore, this Aspergillus flocculosus EU NRC strain is of interest for further studies.

\section{Conclusion}

The reported fungus could be used for industrial production of dextranase as it is a non-pathogenic strain which grows at mild conditions and produces extracellular, highly substrate-specific dextranase for the removal of dextran contamination. In this work, Plackett-Burman design was used to determine the relative importance of medium conditions for dextranase production. Among the variables, dextran, medium volume and inoculum age were found to be the most significant variables. From further optimization studies, the optimized values of the variables for maximum dextranase production were as follows: dextran concentration of $2.09 \mathrm{~g} / \mathrm{l}$, medium volume of $52.5 / 250 \mathrm{ml}$ flask and inoculum age of $80.5 \mathrm{~h}$. The maximum production of dextranase was obtained under the optimized media $440(\mathrm{U} / \mathrm{ml})$. The results show a close concordance between the predicted and the experimental run. These results may provide an important basis for industrial applications.

Acknowledgments The authors gratefully acknowledge the financial assistance from the Department of Chemistry of Natural and Microbial Products at the National Research Center.

Conflict of interest We declare that we have no conflict of interest. 
Open Access This article is distributed under the terms of the Creative Commons Attribution License which permits any use, distribution, and reproduction in any medium, provided the original author(s) and the source are credited.

\section{References}

Abdel-Naby MA, Ismail AS, Abdel-Fattah AM, Abdel- Fattah AM (1999) Preparation and some properties of immobilized Penicillium funiculosum 258 dextranase. Process Biochem 34:391

Akhnazarova S, Kafarov V (1982) Experiment optimization in chemistry and chemical engineering. Mir publications, Moscow, Chicago, pp 245-262

Barrett JF, Curtiss RIII (1986) Renaturation of dextranase activity from culture supernatant of Streptococcus sobrinus after sodium dodecylsulfate polyacrylamide gel electrophoresis. Anal Biochem 158:365-370

Box GEP, Behnken DW (1960) Some new three level designs for the study of quantitative variables. Technometrics 2:455-475

Box GEP, Wilson KB (1951) On the experimental attainment of optimum conditions. J R Stat Soc Ser B Methodol 13:1-45

Box GEP, Hunter WH, Hunter IS (1978) An introduction to design data analysis and model building. Wiley, London

Chu JN, Young CC, Tan CC, Wu SP, Young LS (2012) Improvement of productivity and polysaccharide-protein complex in Agaricus blazei. Pesquisa Agropecuária Brasileira 47:96-102

Davis RS, Habra L, Isenberg DL (1974) Method of producing dextranase. US Patent 3, 787, 289

Eggleston G, Monge A (2004) Optimization of sugarcane factory application of commercial dextranases. Process Biochem 40:1881

Fukumoto J, Tsuji H, Tsuru D (1971) Studies on mold dextranases 1. Penicillium luteum dextranase: its production and some enzymatic properties. J Biochem 69:1113

Fulcher R, Inkerman PA (1974) Proc Queensland Sugar Cane Technol 43:295

Han Y, Li ZY, Miao XL, Zhang FL (2008) Statistical optimization of medium components to improve the chitinase activity of Streptomyces sp. Da11 associated with the South China Sea sponge Craniella australiensis. Process Biochem 43:1088-1093

Hattori A, Ishibashi K (1981) Screening of dextranase producing microorganisms. Agricult Biol Chem 45:2347

Hidi S (1975) QSSCT:331-343

Hiraoka N, Fukumoto J, Tsuru D (1972) Studies on mold dextranases: 3. Purification and some enzymatic properties of Aspergillus carneus dextranase. J Biochem 71:57

Janson JC, Porath J (1966) A bacterial dextranase. Methods Enzymol 8:615-621

Kar S, Swain MR, Ray RC (2009) Statistical optimization of alphaamylase production with immobilized cells of Streptomyces erumpens MTCC 7317 in Luffa cylindrical L. sponge. Appl Biochem Biotechnol 152:177-188

Khalikova EF, Susi P, Usanov NG, Korpela T (2005) Microbial dextran- hydrolyzing enzymes: fundamentals and applications. Microbiol Mol Biol Rev 69:306-325

Khuri AI, Cornell JA (1987) Response surfaces: designs and analyses. Marcel Dekker Inc, New York

Levine R (2005) Finance and growth: theory, evidence and mechanisms. In: Aghion P, Durlauf S (eds) Handbook of economic growth, vol 1, part 1. North Holland, Amsterdam, pp 865-934

Madhu PKA (1984) Studies on dextranase from Penicillium aculeatum. Enzym Microb Technol 6:217

Marotta M, Martino A, De Rosa A, Farina E, Carteni M, De Rosa M (2002) Degradation of dental plaque glucans and prevention of glucan formation using commercial enzymes. Process Biochem 38:101

Mghir AS, Cremieux AC, Jambou R, Muffat-Jolly M, Pocidalo JJ, Carbon C (1994) Dextranase enhances antibiotic efficacy in experimental viridans streptococcal endocarditis. Antimicrob Agent Chemother 38:953

Molodova GA, Danilova N, Maksimov VI, Kozhevnikova NP (1980) Methods of purifying dextranase from Penicillium fimiculosum. Prikl Microbiol 16:907-914

Muralidhar RV, Chirumamila RR, Marchant R, Nigam P (2001) A response surface approach for the comparison of lipase production by Candida cylindracea using two different carbon sources. Biochem Eng 9:17-23

Myers RH, Montgomery CM (1995) Response surfaces methodology: process and product optimization using designed experiments. Wiley, New York

Niladevi KN, Sukumaran RK, Jacob N, Anisha GS, Prema P (2009) Optimization of laccase production from a novel strain-Streptomyces psammoticus using response surface methodology. Microbiol Res 164:105-113

Novo Nordisk A/S (1977) Dextranase Novo 25L. A dextran decomposing enzyme for the sugar industry. Product data information 112-GB, Novo Enzyme Division, Dagsvaerd, Denmark

Plackett RL, Burman JP (1946) The design of optimum multifactorial experiments. Biometrika 33:305-325

Pleszczynska M, Szczodrak J, Rogalski J, Fiedurek J (1997) Hydrolysis of dextran by Penicillium notatum dextranase and identification of final digestion products. Mycol Res 101:69

Priyanka J, Santosh K (2011) Impact of media on isolation of dextranase producing fungal strains. J Sci Res 55:71-76

Salihu A, Alam MZ, AbdulKarim MI, Salleh HM (2011) Optimization of lipase production by Candida cylindracea in palm oil mill effluent based medium using statistical experimental design. J Mol Catal Enzym 69:66-73

Sankpal NV, Joshi AP, Sainkar AP, Kulkarni BD (2001) Production of dextran by Rhizopus sp. Immobilized on porous cellulose support. Process Biochem 37:395-403

Sastry SV, Khan MA (1998) Aqueous based polymeric dispersion: Plackett-Burman design for screening of formulation variables of atenolol gastrointestinal therapeutic system. Pharm Acta Helv 73:105-112

Shukla GL, Madhu PKA (1989) Study of some parameters for the production of dextranase by Penicillium aculeatum. Enzym Microb Technol 11:533

Simonson LG, Liberta AE, Richardson A (1975) Characterization of an extracellular dextranase from Fusarium moniliforme. Appl Microbiol 30:855

Sreekumar G, Krishnan S (2010) Enhanced biomass production study on probiotic Bacillus subtilis SK09 by medium optimization using response surface methodology. Afr J Biotechnol 9:8078-8084

Thompson JD, Higgins DG, Gibson TJ (1994) CLUSTAL W: improving the sensitivity of progressive multiple sequence alignment through sequence weighting, positions-specific gap penalties and weight matrix choice. Nucleic Acids Res 22:4673-4680

Zhang HZ, Zhang FL, Li ZY (2009) Gene analysis, optimized production and property of marine lipase from Bacillus pumilus B106 associated with South China Sea sponge Halichondria rugosa. World J Microbiol Biotechnol 25:1267-1274

Zheng Z, Scott S, Lukas W, Miller Webb (2000) A greedy algorithm for aligning DNA sequences. J Comput Biol 7:203-214

Zhihong W, Yoshihiko T, Göran B, Xiao-Ru W (2003) 18S rRNA Gene Variation among Common Airborne Fungi, and Development of Specific Oligonucleotide Probes for the Detection of Fungal Isolates. Appl Environ Microbiol 69:5389-5397 